Solid State Ionics 11 (1984) 285-291

North-Holland, Amsterdam

\title{
BULK CONDUCTIVITY AND POLARIZATION OF IONIC CRYSTALS EXHIBITING DEFECT EXCHANGE BETWEEN INEQUIVALENT SITES
}

\author{
Donald R. FRANCESCHETTI is \\ Solid State Department, Physics Laboratory, State University, \\ P.O. Box 80000,3508 TA Utrecht, The Netherlands \\ and \\ Paul C. SHIPE \\ Department of Physics, Memphis State University, Memphis, Tennessee 38152, USA
}

Received 3 December 1982

Revised manuscript received 31 May 1983

\begin{abstract}
The bulk conductivity of ionically conducting crystals in which Schottky or Frenkel defects jump between inequivalent sites can be appreciably more complex than that of simpler materials. An equivalent electrical circuit is derived to describe the bulk ac response of crystals of the tysonite type. The circuit has the form of a two-component Maxwell-Wagner capacitor and includes a capacitative element which represents a frequency-dependent bulk polarization not associated with dipolar complexes. The application of the model to $\mathrm{LaF}_{3}$ is discussed.
\end{abstract}

\section{Introduction}

The bulk ionic conductivity of simple crystals with small concentrations of Schottky or Frenkel defects is now well understood. Elementary treatments of the ionic conductivity of solids $[1,2]$ usually include a derivation of the bulk conductivity for materials in which the motion of each charge-carrying species is governed by a single, thermally activated, jump probability. In crystals with moderately complex lattice structures, however, the motion of a given defect species may involve several distinguishable jump processes, each governed by a different activation enthalpy.

In connection with recent experimental studies of the small-signal ac response of $\mathrm{LaF}_{3}[3,4]$, the present authors have developed a generalization of the previous simple theory $[1,2]$, which takes into account the existence of several different possible jump processes for anion vacancies in materials of the tysonite structure. Our treatment leads to a number of interesting predictions which should also apply to some other groups of ionically conducting solids. We have found that the bulk ac response of such materials can include a frequency-dependent polarization which mimics the effect of reorientable bound dipolar complexes and a bulk conductivity which, as a function of temperature, can mimic the dissociation of bound dipoles or the transition from extrinsic to intrinsic conduction. In the present paper we present our model for the small-signal ac bulk response of tysonite-type materials, derive an equivalent circuit for the bulk admittance, compare our predictions with some of the experimental data for LaF 3 and its solid solutions, and comment briefly on the applicability of our predictions to other materials.

\footnotetext{
${ }^{4}$ Permanent address: Department of Physics, Memphis State University, Memphis, Tennessee 38152, USA.
}

0 167-2738/84/\$ 03.00 e Elsevier Science Publishers B.V.

(North-Holland Physics Publishing Division) 


\section{Model and equivalent circuit}

The model employed in our treatment of tysonite-type materials is illustrated in fig. 1. We consider a single species of mobile charge-carrying defect which can occupy sites on either of two different sublattices, A and B, and assume that the applied alternating electric field is in a direction such that lattice planes perpendicular to this direction contain sites of the A sublattice or B sublattice only. The sequence of lattice planes encountered in the direction of the applied field is AABAAB... . Migration of the charged defect species will involve jumps from one A lattice plane to its neighboring lattice plane and between $A$ and $B$ lattice planes or from one A lattice plane to another A lattice plane passing through a B lattice plane.

We consider a group of three lattice planes in the bulk of the crystal in the sequence AAB at right angles to a weak externally applied alternating electric field with spatially averaged intensity $E_{0} \exp (\mathrm{i} \omega t)$. We let $n_{10}, n_{20}$ and $n_{30}$ respectively denote the equilibrium number of defects (per unit area) on the first A plane, the second A plane and the $B$ plane. The deviations from the equilibrium concentrations will be denoted $n_{i 1} \exp (i \omega t)$. We define the field deviations $E_{i j}(j=i \pm 1)$ such that the average electric field between the $i$ th and $j$ th planes is $\left(E_{0}+E_{i j}\right)$ $\mathrm{X} \exp (\mathrm{i} \omega t)$. We further define the thermal equilibrium jump probabilities $P_{0}$, between nearest-neighbor $\mathrm{A}$ planes, $P_{1}$, from the B plane to its nearest-neighbor A plane, $P_{2}$, from the A plane to its nearest-neighbor B plane, and $P_{3}$, from one A plane passing through the nearest-neighbor $B$ plane to the next $A$ plane.

The variables just defined are subject to a number of important constraints. Thermal equilibrium requires that $n_{20}=n_{10}$ and $n_{30}=\left(P_{2} / P_{1}\right) n_{10}$. It then follows that the number of defects per unit volume, $c_{0}$, is given by

$$
c_{0}=\left(2+P_{2} / P_{1}\right) n_{10} /(a+2 b) \text {, }
$$

where $a$ and $b$ are the distances indicated in fig. 1. If we treat the mobile charge carriers as point charges, it follows from Gauss's law and translational invariance that $E_{12}-E_{31}=\beta n_{11}, E_{23}-E_{12}=\beta n_{21}$ and $E_{31}-E_{23}=\beta n_{31}$, where $\beta \equiv 4 \pi e / \epsilon$, and $\epsilon$ is the bulk low-frequency dielectric constant of the electrolyte appropriate to the direction of the applied electric field $\left(\epsilon_{\perp}\right.$ or $\left.\epsilon_{\|}\right)$. Bulk electroneutrality provides the important condition

$$
n_{11}+n_{21}+n_{31}=0
$$

while defining $E_{0} \exp (i \omega t)$ as the spatially averaged electric field requires that $a E_{12}+b E_{23}+b E_{31}=0$. On combining this last.condition with the Gauss's law relations, one obtains expressions for the $E_{i j}$ in terms of the concentration perturbations, namely

$$
\begin{aligned}
& E_{12}=\beta b\left(n_{11}-n_{21}\right) /(a+2 b), \\
& E_{23}=\beta\left(a n_{21}-b n_{31}\right) /(a+2 b),
\end{aligned}
$$

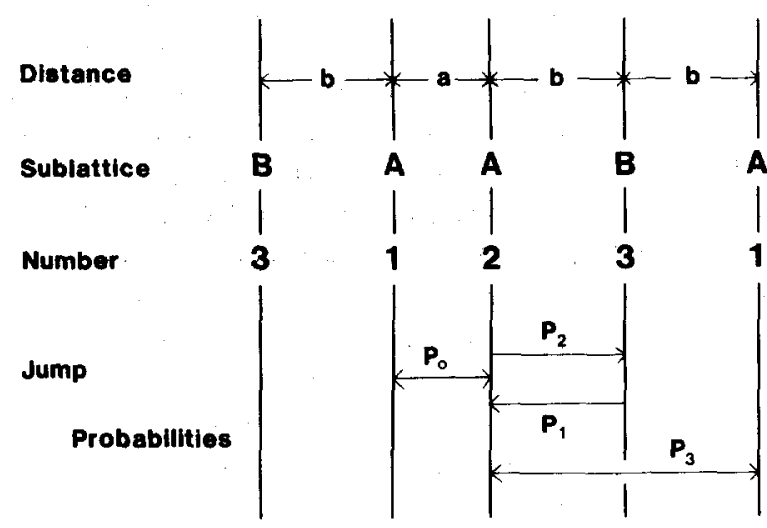

Fig. 1. Model used in calculating bulk ac response of tysonitetype materials. Vertical lines indicate lattice planes. Applied electric field is directed horizontally. 
and

$$
E_{31}=\beta\left(b n_{31}-a n_{11}\right) /(a+2 b) .
$$

We assume that each of the jump probabilities $P_{i}$ has the usual thermally activated form and that in each case the energy barrier has its maximum at the point midway between initial and final lattice planes. Thus, in the presence of a local electric field $E$ the jump probability becomes, approximately, $P_{i} \exp ( \pm E d / 2 k T)$, where $d$ is the distance of the jump, and $P_{i}=P_{i 0} \exp \left(-\Delta H_{i} / k T\right)$, where $\Delta H_{i}$ is the activation enthalpy for the jump. We therefore have for the fluxes $J_{i j} \exp (i \omega t)$, of charge carriers between the $i$ th and $j=(i \pm 1)$ st lattice planes,

$$
\begin{aligned}
& J_{12} \exp (\mathrm{i} \omega t)=\left[n_{10}+n_{11} \exp (\mathrm{i} \omega t)\right] P_{0} \exp \left[e\left(E_{0}+E_{12}\right) \exp (\mathrm{i} \omega t) a / 2 k T\right] \\
& -\left[n_{20}+n_{21} \exp (\mathrm{i} \omega t)\right] P_{0} \exp \left[-e\left(E_{0}+E_{12}\right) \exp (\mathrm{i} \omega t) a / 2 k T\right], \\
& J_{23} \exp (\mathrm{i} \omega t)=\left[n_{20}+n_{21} \exp (\mathrm{i} \omega t)\right] P_{2} \exp \left[e\left(E_{0}+E_{23}\right) b \exp (\mathrm{i} \omega t) / 2 k T\right] \\
& -\left[n_{30}+n_{31} \exp (\mathrm{i} \omega t)\right] P_{1} \exp \left[-e\left(E_{0}+E_{23}\right) b \exp (\mathrm{i} \omega t) / 2 k T\right] \\
& +\left[n_{20}+n_{21} \exp (\mathrm{i} \omega t)\right] P_{3} \exp \left[e\left(E_{0}+E_{23}\right) b \exp (\mathrm{i} \omega t) / k T\right] \\
& -\left[n_{10}+n_{11} \exp (\mathrm{i} \omega t)\right] P_{3} \exp \left[-e\left(E_{0}+E_{31}\right) b \exp (\mathrm{i} \omega t) / k T\right],
\end{aligned}
$$

and

$$
\begin{gathered}
J_{31} \exp (\mathrm{i} \omega t)=\left[n_{30}+n_{31} \exp (\mathrm{i} \omega t)\right] P_{1} \exp \left[e\left(E_{0}+E_{31}\right) b \exp (\mathrm{i} \omega t) / 2 k T\right] \\
-\left[n_{10}+n_{11} \exp (\mathrm{i} \omega t)\right] P_{2} \exp \left[-e\left(E_{0}+E_{31}\right) b \exp (\mathrm{i} \omega t) / 2 k T\right] \\
+\left[n_{20}+n_{21} \exp (\mathrm{i} \omega t)\right] P_{3} \exp \left[e\left(E_{0}+E_{23}\right) b \exp (\mathrm{i} \omega t) / k T\right] \\
-\left[n_{10}+n_{11} \exp (\mathrm{i} \omega t)\right] P_{3} \exp \left[-e\left(E_{0}+E_{31}\right) b \exp (\mathrm{i} \omega t) / k T\right] .
\end{gathered}
$$

The total current $I \exp (i \omega t)$ is spatially invariant [5] and can be expressed as the sum of faradaic and displacement currents.

$$
I \exp (\mathrm{i} \omega t)=e J_{i j} \exp (\mathrm{i} \omega t)+(\mathrm{i} \omega \epsilon / 4 \pi)\left(E_{0}+E_{i j}\right) \exp (\mathrm{i} \omega t) .
$$

Solution of the preceding equations to find $I$ in terms of $E_{0}$ is straightforward but rather tedious. First one linearizes eqs. (6)-(9) by expanding the exponential Boltzmann factors and dropping terms higher than first order in $E_{0}, E_{i j}$ or $\exp (i \omega t)$. Next one uses eqs. (3)-(5) in the linearized equations and in eq. (9) to eliminate the $E_{i j}$. By inserting each of the linearized forms of eqs. (6)-(8) in eq. (9) one obtains three equations relating $I$ to the $n_{i 1}$. These three equations, together with eq. (2) can then be solved simultaneously for the four unknowns $I$ and $n_{i 1}$ $(i=1,2,3)$ in terms of $E_{0}$.

For a sample thickness $l$, the system admittance $Y \equiv I / V=I / E_{0} l$ is given exactly by the circuit of fig. 2, with circuit elements (per unit cross-sectional area) given by

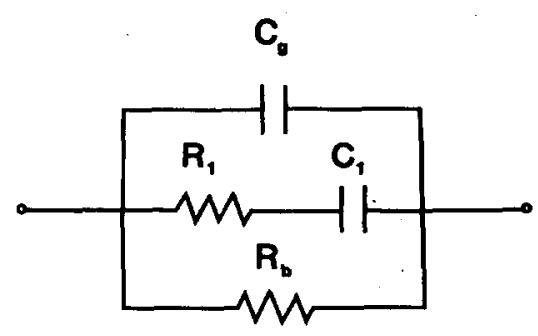

Fig. 2. Equivalent circuit for bulk small-signal ac response of tysonite-type materials. 


$$
\begin{aligned}
& C_{\mathrm{g}}=\epsilon / 4 \pi l . \\
& R_{1}=\frac{l k T}{e^{2} c_{0}} \frac{\left(P_{2}+2 P_{3}+2 P_{0}\right)\left(2+P_{2} / P_{1}\right)}{\left(P_{2}+2 P_{3}\right)\left[2 b^{2}\left(P_{2}+2 P_{3}\right)-4 a b P_{0}\right]+2 P_{0}^{2} a^{2}}, \\
& C_{1}=\frac{e^{2} c_{0}}{l k T} \frac{\left.\left\{P_{2}+2 P_{3}\right)\left[2 b^{2}\left(P_{2}+2 P_{3}\right)-4 a b P_{0}\right]+2 P_{0}^{2} a^{2}\right\}}{\left(2+P_{2} / P_{1}+a b / L L_{\mathrm{D} 1}^{2}\right)\left(P_{2}+2 P_{3}+2 P_{0}\right)^{2}},
\end{aligned}
$$

and

$$
R_{\mathrm{b}}=\frac{l k T}{e^{2} c_{0}} \frac{\left(P_{2}+2 P_{3}+2 P_{0}\right)\left(2+P_{2} / P_{1}\right)}{P_{0}\left(P_{2}+2 P_{3}\right)(a+2 b)^{2}}
$$

where

$$
L_{\mathrm{D} 1} \equiv \epsilon k T / 4 \pi e^{2} c_{0},
$$

is the one-mobile Debye length of the material.

$C_{\mathrm{g}}$ is the geometric capacitance of the sample and $R_{\mathrm{b}}$ its limiting low-frequency bulk resistance. The elements $R_{1}$ and $C_{1}$ describe the behavior of a macroscopic bulk polarization arising from the inequality of the jump probabilities. If we set $P_{3}=0, a=b$, and $P=P_{1}=P_{2}$ so that all nearest-neighbor jumps are equivalent, we find $R_{1} \rightarrow \infty$, $C_{1} \rightarrow 0$ and $R_{\mathrm{b}} \rightarrow R_{\mathrm{b} 0}$, with

$$
R_{\mathrm{b} 0}=l k T / e^{2} c_{0} P_{0} a^{2},
$$

so that the equivalent circuit reduces to the geometric capacitance and bulk resistance in parallel. The appearance of the Debye length in the expression for $C_{1}$ is consistent with a polarization arising from the motion of free charge carriers, and represents the screening of the internal field by the mobile charges.

The equivalent circuit is identical to that of a two-component Maxwell-Wagner capacitor [6,7], in which alternating layers of two different lossy dielectrics are present. With the exception of $R_{\mathrm{b}}$ it is identical to the circuit derived by Debye $[8,9]$ for a medium with reorientable permanent dipoles. The circuit parameters in the present case may, however, exhibit a rather different temperature dependence than in the Debye model. For a solid with bound dipolar defect pairs one expects $C_{1}$ to decrease with increasing temperature, as the dipolar pairs dissociate, while in the present case $C_{1}$ may increase or decrease with temperature, depending on the free energies of defect formation and the activation enthalpies associated with the $P_{i}$. For intrinsic materials it is probable that the dominant effect would be the increase in $c_{0}$ with increasing temperature and that $C_{1}$ would correspondingly increase.

$R_{\mathrm{b}}$ is the bulk resistance which would be observed at zero frequency in the absence of electrode polarization. The time constant for the middle branch of the equivalent circuit is

$$
\tau_{1} \equiv R_{1} C_{1}=\frac{2+P_{2} / P_{1}}{\left(2+P_{2} / P_{1}+a b / L_{\mathrm{D} 1}^{2}\right)\left(P_{2}+2 P_{3}+2 P_{0}\right)}
$$

At frequencies much greater than $\tau_{1}^{-1}$, the apparent bulk resistance will be the parallel combination of $R_{1}$ and $R_{\mathrm{b}}$, that is

$$
R_{\mathrm{P}}=\frac{l k T}{e^{2} c_{0}} \frac{\left(2+P_{2} / P_{1}\right)}{a^{2} P_{0}+2 b^{2}\left(P_{2}+2 P_{3}\right)} .
$$




\section{Application to $\mathrm{LaF}_{3}$}

The dominant disorder in $\mathrm{LaF}_{3}$ is believed to be of the Schottky type

$$
\mathrm{O} \rightleftharpoons \mathrm{V}_{\mathrm{La}}^{\prime \prime \prime}+3 \mathrm{~V}_{\mathrm{F}}^{\bullet}
$$

with the cation vacancies essentially immobile so that the ionic current is carried by the anion vacancies alone. Although there is still some disagreement concerning the exact lattice structure of $\mathrm{LaF}_{3}[10]$, the weight of recent evidence [11-13] favors a trigonal structure of space group $P \overline{3} c 1-D_{3 d}^{4}$, in which there are three distinct fluoride sublattices $\alpha, \beta$ and $\gamma$ sites in the ratio $12: 4: 2$. The $\beta$ and $\gamma$ sites are, however, almost identical in environment and cannot be distinguished in NMR experiments [12]. In examining a model of the $\mathrm{LaF}_{3}$ (tysonite) structure one finds that in the direction of the $c$ axis, and in the direction of the bisector of the $a$ and $b$ axes, the lattice planes occur in the pattern shown in fig. 1 , with the A planes containing only $\alpha$-type fluoride sites and the B planes containing $\beta$ - and $\gamma$-type sites.

The electrical properties of $\mathrm{LaF}_{3}$ are still the subject of some disagreement [14]. This is at least partly due to the tendency of $\mathrm{LaF}_{3}$ crystals to form oxygen-rich surface layers, which interfere with the determination of the bulk electrical properties. Experimental plots $[14,15]$ of $\log \sigma T$ against $1 / T$ show a change in slope at about $100^{\circ} \mathrm{C}$ for the pure single crystal and dilute solid solutions $\mathrm{La}_{1-x} \mathrm{Ba}_{x} \mathrm{~F}_{3-x}$. Sher et al. [15] interpret the change in slope as due to the dissociation of impurity-vacancy pairs, with the impurity being either oxygen or a divalent cation. Schoonman et al. [14], however, discount this interpretation on the basis of their measurements on the solid solution up to $x=0.05$. As we shall see, a comparable $\log \sigma T$ versus $1 / T$ plot can be obtained from the present model, without invoking the dissociation of impurity-vacancy pairs.

An estimate of the pre-exponential factor and activation enthalpies for the jump probabilities can be obtained by fitting conductivity,

$$
\sigma=e^{2} c_{0} P_{0} a^{2} / k T,
$$

obtained by assuming all nearest-neighbor jumps to be equivalent (see eq. (15)), to the low-temperature solid solution data of Schoonman et al. [14]. Taking $a=2.40 \AA$ and fitting to the $x=0.01$ curve yields $P_{00}=2.45 \times 10^{15}$ $\mathrm{s}^{-1}$ and $\Delta H_{0}=0.49 \mathrm{eV}$.

The reported bulk conductivities are obtained by extrapolation of the high-frequency admittance data. Thus if the present model is applicable they would correspond to the parallel resistance $R_{\mathrm{p}}$ of eq. (17). We have examined in detail the temperature dependence of $R_{\mathrm{P}}$, and the corresponding $\sigma$ as calculated for the dilute solid solution $\mathrm{La}_{1-x} \mathrm{Ba}_{x} \mathrm{~F}_{3-x}(x=0.01)$ using the $P_{00}$ and $\Delta H_{i}(i=1,2,3)$ values in the ranges $10^{6}-10^{12} \mathrm{~s}^{-1}$ and $0.08-0.78$ $\mathrm{eV}$, respectively. In our calculations we assume a temperature-independent concentration of fluoride vacancies and set $a=b=2.40 \AA$. Some representative results for cases in which $P_{3}=0$ are shown in fig. 3 . As can be seen in this figure, the $\log \sigma T$ versus $10^{3} / T$ plot can exhibit a downward "knee" (as in curve $a$ ), or an upward "knee" (as in curve $c$ ), or relatively linear behavior. Choosing non-zero values for $P_{30}$ and $\Delta H_{3}$ can produce relatively little change in the $\log \sigma T$ versus $10^{3} / T$ plot or can introduce an additional "knee" or can change the character of the "knee" already present.

Under extrinsic conditions ( $c_{0}$ independent of temperature) the capacitance $C_{1}$ is found to be a generally decreasing function of $T$, although for certain parameter choices $C_{1}$ will increase with increasing temperature over a limited temperature range. Under intrinsic conditions the increase of $c_{0}$ with increasing temperature can lead to a general increase in $C_{1}$.

Of the many theoretical conductivity curves examined, the curve labelled "a" in fig. 3 was found to come closest to the data of ref. [14], displaying a change in slope at about $T=120^{\circ} \mathrm{C}$. It must be noted, however, that the parameter values for this curve lead to a $C_{1}$ which decreases monotonically with increasing temperature, al though the equivalent-circuit analysis of the ac data [4] indicates the opposite effect. Nonetheless, the other two preexponential factors and activation enthalpies obtained by our trial-and-error procedure are in rough agreement with the values obtained by Jaroszkiewicz and Strange [12] from the analysis of ${ }^{19} \mathrm{~F}$ NMR relaxation times. It 


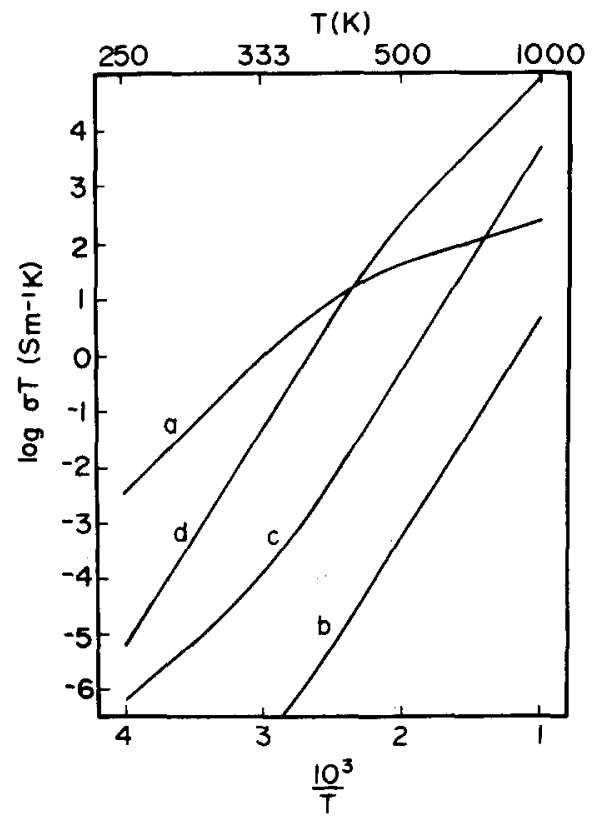

Fig. 3. High-frequency bulk conductivity for $\mathrm{La}_{1-x} \mathrm{Ba}_{x} \mathrm{~F}_{3-x}$ $(x=0.01)$ computed from $R_{\mathrm{P}}$ (eq. (17)). $P_{00}=2.45 \times 10^{15}$ $\mathrm{s}^{-1}, \Delta H_{0}=0.49 \mathrm{eV}$ and $P_{30}=0$ for all curves. Curve a: $P_{10}$ $=10^{6} \mathrm{~s}^{-1}, \Delta H_{1}=0.25 \mathrm{eV}, P_{20}=4 \times 10^{10} \mathrm{~s}^{-1}, \Delta H_{2}=0.60 \mathrm{eV}$. Curve b: $P_{10}=10^{6} \mathrm{~s}^{-1}, \Delta H_{1}=0.39 \mathrm{eV}, P_{20}=10^{9} \mathrm{~s}^{-1}, \Delta H_{2}$ $=0.39 \mathrm{eV}$. Curve c: $P_{10}=10^{9} \mathrm{~s}^{-1}, \Delta H_{1}=0.39 \mathrm{eV}, P_{20}=10^{9}$ $\mathrm{s}^{-1}, \Delta H_{2}=0.09 \mathrm{eV}$. Curve d: $P_{10}=10^{9} \mathrm{~s}^{-1}, \Delta H_{1}=0.39 \mathrm{eV}$, $P_{20}=10^{6} \mathrm{~s}^{-1}, \Delta H_{2}=0.09 \mathrm{eV}$.

seems reasonable that the actual situation in the bulk of $\mathrm{LaF}_{3}$ may be somewhat more complicated than that envisioned here, possibly owing to the presence of some residual bound dipolar complexes, or cooperative interactions between charge carriers. The ability of the present model to rationalize both the form of the equivalent circuit and the qualitative form of the $\log \sigma T$ plot suggest, however, that its basic features will need to be included in any more complete analysis.

\section{Applicability to other materials}

Although our analysis was based on a simplified version of the tysonite structure, it may readily be argued that similar effects may be encountered in other reasonably complex crystal structures. Whenever the migration of a charge-carrying species involves a series of inequivalent jumps, one may expect a bulk resistivity characterized by more than one activation enthalpy and a capacitative component to the bulk response, even in the absence of bound dipolar complexes. Our results suggest that in general the bulk response may be modelled by an $n$-component Maxwell-Wagner type of equivalent circuit, possibly with $n>2$ in rather complex lattice structures. If the existence of inequivalent jump processes is overlooked in the analysis of ac response data, some serious misinterpretations are possible.

\section{Acknowledgements}

This work was supported in part by Research Corporation Grant No. 9322 and by NATO Reșearch Grant No. 1696. Access to computer graphics facilities provided under US National Science Foundation Grant TFI-8021957 is also gratefully acknowledged. The authors thank Dr. J. Schoonman and A. Roos for helpful discussions and access to experimental data. 


\section{References}

[1] N.F. Mott and R.W. Gurney, Electronic processes in ionic crystals (Oxford, London, 1940).

[2] A.B. Lidiard, Handbuch der Physik 20 (1957) 246.

[3] A. Roos, D.R. Franceschetti, M. Buijs and J. Schoonman, in: Solid state chemistry, eds. R. Metselaar, H.J.M. Heijligers and J. Schoonman (Elsevier, Amsterdam, 1982).

[4] A. Roos, D.R. Franceschetti and J. Schoonman, Solid State Ionics 12 (1984), to be published.

[5] J.R. Macdonald, J. Appl. Phys. 46 (1975) 4602.

[6] A.R. von Hippel, Dielectrics and waves (M.I.T. Press, Cambridge, MA, 1954).

[7] J.R. Macdonald, in: Electrode processes in solid state ionics, eds. M. Kleitz and J. Dupuy (Reidel, Dordrecht, 1975).

[8] P. Debye, Polar molecules (Chemical Catalogue Company, New York, 1929).

[9] H. Frölich, Theory of dielectrics (Oxford, London, 1958).

[10] Gmelin Handbuch der anorganischen Chemie, Seltenerdelemente, Teil C3. Sc, Y, La and Lanthanide, 8th Ed. (Springer, Berlin, 1976) pp. 50ff.

[11] A. Zalkin, D.H. Templeton and T.E. Hopkins, Inorg. Chem. 5 (1966) 1466.

[12] G.A. Jaroszkiewicz and J.H. Strange, J. Phys. Coll. C6 (1980) 246.

[13] A.K. Cheetham, private communication.

[14] J. Schoonman, G. Oversluizen and K.E.D. Wapenaar, Solid State Ionics 1 (1980) 211.

[15] A. Sher, R. Solomon, K. Lee and M.W. Muller, Phys. Rev. 144 (1966) 593. 